Africa Development, Vol. XXV, Nos. $1 \& 2,2000$

\title{
La libéralisation financière internationale et la crise du développement est-asiatique
}

\author{
Jomo Kwame Sundaram *
}

Résumé : Le présent article explore les causes profondes de la crise financière de 1997 qui a frappé le monde est-asiatique et dévalué les monnaies et le pouvoir économique des nouveaux pays industriels de la seconde génération. Il affirme que nonobstant l'existence, autrefois, d'une telle crise dans la même région, la réponse appropriée au problème actuel se trouve ailleurs. D' autre part, comme le marché, qui est de plus en plus livré à luì-mème, n'a ni la mémoire ni la capacité d'acquérir une immunité naturelle, il revient donc aux responsables d'élaboration des politiques d'établir les institutions nécessaires de la gestion des affaires publiques.

Abstract: This article critically explores the roots of the financial crisis of 1997 that tormented the East-Asian region and devalued the currencies and economic power of the second generation newly industrialised countries. It argues that despite the existence of such crisis in the same region in the past, the appropriate response to current problem lies elsewhere. Given that the market, which has become more and more self governing, lacks memory and the capacity to be naturally immuned, the onus is on the actors to elaborate policies to establish the necessary institutions and reconsider and restructure the institutional mechanisms for the management of public affairs.

Après des mois d'attaques spéculatives contre le baht thailandais, la Banque de Thaillande a laissé flotter sa monnaie à partir du 2 juillet 1997.

* Je tiens à témoigner ma reconnaissance à Jan Kregel, Al Alim Ibrahim, Din Merican et Warren Bailey pour m'avoir formulé des observations critiques tiles, qui ne les engagent toutefois en aucune façon. Les principales causes et conséquences des récentes crises monétaires et financières en Asie du Sud-Est, surtout en Malaisie, continuent de susciter un débat considérable. Le présent essai se veut polémique, puisqu'il n'existe à l'évidence aucun consensus sur les diverses questions controversées en jeu. Autant que faire se peut, le langage utilisé n'est pas technique, de manière à être accessible à un lectorat aussi vaste que possible. Puisque la situation continue d'évoluer, ces réflexions sont sujettes à révision au fil du temps, des événements et des tendances. Les critiques et suggestions sont done particulièrement appréciées. 


\section{Africa Development, Vol. XXV, Nos. 1 \& 2, 2000}

Après des mois d'attaques spéculatives contre le baht thaillandais, la Banque de Thaillande a laissé flotter sa monnaie à partir du 2 juillet 1997. A la mijuillet, les monnaies des trois nouveaux pays industriels (NPI) de deuxième génération situés en Asie du Sud-Est, c'est-à-dire celles aussi de l'Indonésie et de la Malaisie, avaient brusquement chuté, leurs marchés boursiers suivant ce mouvement. Avant la fin de 1997, en dépit de sa structure économique assez différente, la Corée du Sud s'est elle aussi effondrée. La plupart des autres économies d'Asie du Sud-Est ont également subi des pressions considérables, directement (dans le cas de l'attaque contre le dollar de Hong Kong) ou indirectement (en vue de conserver un avantage comparatif de coût par rapport aux monnaies désormais fort dépréciées des pays exportateurs sud-asiatiques).

Contrairement à l'impression donnée par un grand nombre de journalistes et de commentateurs économiques, ainsi que par le Fonds monétaire international (FMI), les causes et les caractéristiques de la crise sont encore loin de faire l'unanimité. En effet, il n'est qu'à constater le débat engagé entre le FMI et ses détracteurs sur le bien-fondé de ses programmes négociés en Thaïlande, en Indonésie et en Corée du Sud. Si les débats d'orientation ont à juste titre particulièrement retenu l'attention, notamment celle du grand public, les crises est-asiatiques ont aussi donné matière à réflexion aux économistes internationaux.

De l' avis de certains, la crise serait essentiellement monétaire, bien que sans doute d'un nouveau type, différente de celles qui ont été auparavant imputées à la prodigalité budgétaire ou à l'indiscipline macroéconomique. Dans une perspective légèrement différente, d'autres économistes estiment qu'il s'agit d'une crise de balance des paiements et insistent sur les déficits des transactions courantes qu'enregistrent certaines des économies touchées. Un nombre croissant semble toutefois convenir que la crise, tout d'abord monétaire, est vite devenue une crise financière généralisée, avant de se répercuter sur l'économie réelle, à cause de la liquidité réduite dans le système financier et des effets de la riposte des pouvoirs publics ainsi que d'autres réactions. 
Les répercussions de la crise sur le développement économique font par ailleurs l'objet d'un débat considérable, surtout pour déterminer si l'expérience est-asiatique des trente dernières années offre des leçons et des solutions de développement qui different de celles que préconisent les partisans de la «contre-révolution» contre l'économie du développement. Comme il est maintenant bien connu, dans le cadre de cette réaction néolibérale, la théorie économique du développement et ses préceptes ont été jugés erronés, du fait qu'ils reposaient sur des distorsions de l'économie néoclassique du bien-être exagérant l'ampleur et les effets de la «défaillance du marché» et sous-estimant la probabilité d'une «défaillance de l'État» et de ses conséquences.

D'influents économistes, à la Banque mondiale et ailleurs, ont cité la crise financière est-asiatique pour critiquer le volume East Asian Miracle, publié par la Banque en 1993. Les critiques ont en particulier dénoncé la reconnaissance par l'étude du succès remporté par le «crédit dirigé» et par ce que l'on nomme désormais la «modération financière», qui aurait pour auteur l'actuel premier vice-président et économiste en chef de la Banque, Joseph Stiglitz, qui s'est également inscrit en faux contre les remèdes employés par le FMI dans la crise financière actuelle.

Du fait que la crise du milieu de 1997 a éclaté peu après que Paul Krugman (1994) eut affirmé que la croissance est-asiatique n'était pas soutenable car elle reposait avant tout sur l'accumulation des facteursqui finiraient par connaître des rendements décroissants-et non sur une croissance de la productivité («la transpiration plutôt que l'inspiration»), de nombreux critiques de tous horizons politiques ont vu dans la crise est-asiatique la preuve de l'argument de Krugman ou d'une de ses variantes. Souvent, on constate un certain triomphalisme néolibéral dans les déclarations hâtives annonçant la fin du miracle asiatique, ou dans les jeux de mots sur «le miracle ou la débâcle», les «tigres ou les gros chats riches», etc. 
Dans le même temps, ces dernières années, on a de mieux en mieux reconnu les grandes différences structurelles et systémiques qui séparent les huit pays d'Asie à forte croissance étudiés par la Banque mondiale (1993), à savoir le Japon, la Corée du Sud, Hong Kong, Singapour, la Malaisie, la Thaïlande et l'Indonésie. Ces trois derniers Etats ont été de plus en plus classés parmi les nouveaux pays industriels (NPI) de deuxième génération situés en Asie du Sud-Est, qui possèdent des caractéristiques fort différentes des autres économies mais présentent bien sûr des variantes individuelles. Selon certains avis, pour des raisons diverses, la politique industrielle ou l'intervention sélective de l'Etat a été beaucoup moins judicieuse et efficace dans ces économies. Au contraire, l'Etat est largement intervenu dans d'autres domaines, par suite d'autres considérations ayant moins trait au développement, notamment en Malaisie et en Indonésie (Jomo et al. 1997). Il semble que de telles interventions soient en partie responsables de la vulnérabilité des NPI de deuxième génération situés en Asie du Sud-Est aux facteurs qui ont précipité la crise financière survenue au milieu de 1997 dans la région.

\section{Problèmes macroéconomiques}

La croissance économique et l'évolution structurelle rapides, principalement associées à l'industrialisation de la région, remontent en général au milieu des années 80 . Tandis que les dévaluations effectuées dans les trois pays ainsi que l'assouplissement de règles pesantes contribuaient à créer des conditions propices à la relocalisation, dans ces pays et dans d'autres économies d'Asie du Sud-Est ainsi qu'en Chine, d'installations de production surtout originaires du Japon et de nouveaux pays industriels de première génération tels la Corée du Sud, Taïwan, Hong Kong et Singapour. Cette croissance dynamique a entretenu une industrialisation à vocation exportatrice pendant une bonne partie des années 90, mais est vite allée de pair avec l'essor d'autres industries de transformation, des services et du bâtiment. 
On ne saurait avancer que les fondamentaux étaient sains en Asie du Sud-Est. Bien que la forte croissance ait été soutenue pendant près d'une décennie, durant laquelle, la plupart du temps, les soldes budgétaires étaient solides, l'expansion monétaire non excessive et l'inflation en général maîtrisée, certains autres indices ont été alarmants. La croissance occasionnée par les exportations qu'ont connue les économies d'Asie du Sud-Est depuis la fin des années 80 a été suivie par un vif essor du bâtiment et de l'immobilier, alimenté par le secteur financier qui a privilégié les investissements à court terme, comportant des prêts sur nantissement prisés par les banquiers, plutôt que des investissements plus productifs, mais souvent plus risqués, dans le secteur manufacturier et l'agriculture. L'expansion excessive de l'investissement dans les biens non échangeables a exacerbé les déficits courants. Quoique fréquent en Asie de l'Est, pour diverses raisons, le lien immobilier-finances était particulièrement fort en Thaïlande, ce qui a rendu ce pays plus vulnérable à l'éclatement inévitable de la bulle.

La libéralisation financière engagée à partir des années 80 a par ailleurs eu d'importantes répercussions dans la région, alors que l'épargne étrangère s'ajoutait à l'épargne intérieure déjà élevée. Cette épargne accélère davantage le taux d'accumulation du capital, quoique dans des activités de plus en plus improductives, en raison de la domination étrangère de la plupart des industries soutenant la concurrence internationale. En conséquence, au milieu des années 90 , la croissance rapide observée pendant la dernière décennie a suscité plusieurs préoccupations macroéconomiques apparentées.

Premièrement, l'écart épargne-investissement équivalant à 5 pour cent du PNB en 1997 était à l'origine du déficit des transactions courantes, ${ }^{1}$ qui a dépassé 12 milliards de ringgit malaisiens depuis

'Pendant ce temps, les «analystes financiers» sont devenus si obnubilés par le déficit des transactions courantes que cet indicateur, quasiment à lui seul, est devenu le fétiche des analystes financiers, surtout depuis la crise mexicaine du début de 1995. 
1994. L'écart avait été comblé historiquement par un large recours aux investissements directs étrangers (IDE). Mais l'importance de ces derniers et de la dette extérieure a, à son tour, provoqué un exode croissant des revenus des investissements vers 1'étranger. ${ }^{2}$ Au cours des dernières années en particulier, le déficit courant a été temporairement comblé par des entrées de capitaux à court terme, comme en 1993 et depuis 1995, ce qui a entraîné par la suite des conséquences désastreuses lorsque ces flux se sont inversés. Un grand nombre de mesures récentes de rétablissement de la confiance visent à encourager la reprise des apports de capitaux à court terme, mais ne peuvent résoudre le problème sous-jacent à moyen et à long terme. ${ }^{3}$

Bien que toujours minoritaires, ce sont les institutions de placement à l'étranger qui ont «fait» les marchés boursiers de la région, transférant leurs actifs entre les marchés des valeurs mobilières et les différents types d'options d'investissement financier dans le monde entier. Étant donné leur transparence limitée, le caractère régional de leur présence, la nature des incitations et des rémunérations des gestionnaires de fonds et la brève échéance de

Auparavant, à une époque différente, certaines économies ont enregistré des déficits analogues beaucoup plus prolongés, sans subir de conséquenceśs comparables. Comme on l'a noté immédiatement après la crise mexicaine de 1995, plusieurs économies d'Asie du Sud-Est affichaient alors déjà des déficits comparables aux transactions courantes, malgré la croissance économique rapide, ou plutôt à cause d'elle. Cependant, comme 1'a observé Fisher, les marchés monétaires n'ont pas réussi à s'ajuster plus tôt en Asie du Sud-Est.

${ }^{2}$ Bien entendu, l'accessibilité de capitaux étrangers à bon marché, du fait d'un taux d'intérêt réel peu élevé, peut contribuer temporairement à combler l'écart épargneinvestissement et à pallier la pénurie de devises, en particulier si ces capitaux sont bien investis ou déployés.

${ }^{3}$ A cet égard, il est intéressant de noter que le gouvernement du Chili, influencé par l'Ecole de Chicago, a maintenu de stricts contrôles des mouvements de capitaux. Les investissements de portefeuille au Chili sont autorisés à la Bourse de New York, plutôt qu'à celle de Santiago, alors que contrairement aux investissements directs étrangers, les apports de capitaux au titre des investissements de portefeuille sont soumis à des conditions qui rendent les sorties difficiles. 
leurs investissements, les institutions financières étrangères étaient beaucoup plus enclines à des comportements moutonniers et ce sont elles qui ont le plus contribué à la propagation régionale de la contagion.

Deuxièmement, on a constaté une explosion récente de l'endettement du secteur privé, en particulier envers l'étranger, qui n'est pas sans rapport avec les efforts déployés par les «promoteurs de l'endettement» liés à la croissance des «activités bancaires privées» ${ }^{4}$. Le ratio prêts/PNB a rapidement augmenté au cours des dernières années. Dans le même temps, les engagements extérieurs des banques commerciales ont plus que triplé entre 1995 et 1997. Voilà notamment pourquoi on a tort d'insister sur un relèvement des taux d'intérêt intérieurs, puisque la récente hausse des emprunts contractés par les entreprises provient en grande partie de l'étranger. Ce phénomène a exacerbé les effets de la crise actuelle causant une triple douleur imputable à la dépréciation monétaire, à l'effondrement des marchés boursiers et à la montée des taux d'intérêt.

Pendant ce temps, le surinvestissement de fonds en quête de placement, surtout originaires de l'étranger, n'a fait qu'aggraver la situation, en particulier celle du compte des transactions courantes. Seule une faible proportion des prêts accordés par les banques commerciales et autres s'est dirigée vers le secteur manufacturier, 1'agriculture, les industries extractives et d'autres activités productives; il est probable que ce pourcentage soit encore inférieur dans le cas des emprunts extérieurs, dont la plupart ont été gagés par des actifs tels que des biens immobiliers et des actions. ${ }^{5}$ Autrement

${ }^{4}$ Dans certains pays, les entroprises publiques non financières appartenant à l'État ont largement participé à ce phénomène de croissance de l'endettement, qui concernerait le secteur privé.

${ }^{5}$ Rien ne prouve non plus que l'essor des marchés boursiers observé ces dernières années ait permis de mobiliser plus efficacement des fonds en vue de l'investissement productif; en réalité, le contraire semble plus probable, car pendant 
dit, une bonne part des apports d'épargne étrangère a effectivement contribué à une hausse des prix des actifs, touchant surtout les prix de l'immobilier et des actions. Dans la mesure où de tels investissements n'ont pas concouru à accroître la production de «biens échangeables», ils ont en réalité exacerbé le déficit des transactions courantes, ${ }^{6}$ au lieu de l'atténuer comme on le croyait. Cette détérioration a par la suite aggravé le problème du "déséquilibre des taux de change», alors que les emprunts en dollars américains s'investissaient dans des activités non génératrices de devises. Dans la mesure où une forte proportion de ces emprunts extérieurs était à court terme et déployée pour financer des projets à moyen ou à long terme, un autre problème d' (asymétrie des échéances» s'est aussi posé.

Plus généralement, le risque de change des investissements a souvent augmenté, ce qui a accru la vulnérabilité de ces économies au maintien du quasi-rattachement de leurs monnaies au dollar américain, ${ }^{7}$ régime qui avait par ailleurs favorisé un grand nombre d'emprunts non garantis, qui ont été le fait de membres influents de la société ayant tout intérêt à défendre le taux de change fixe, quels qu'en soient les effets néfastes sur l'économie. À cause de la domination étrangère exercée sur les industries toumées vers les exportations en Asie du Sud-Est, à la différence de l'Asie du NordEst, il n'existait pas de puissants milieux industriels nationaux à vocation exportatrice, capables d'exercer des pressions en faveur de la mise en flottement ou de la dépréciation des monnaies d'Asie du

la dernière décennie, l'intermédiation financière est passée des banques commerciales au marché des valeurs mobilières.

${ }^{6}$ Alors que les économies d'Asie du Sud-Est affichaient des déficits des transactions courantes, les États-Unis se trouvaient dans la même situation, notamment à l'égard de la région, si ce n'est que les conséquences en sont différentes, étant donné le rattachement effectif ou presque au dollar, qui existe à l'heure actuelle dans une grande partie du monde.

${ }^{7}$ Tandis que l'économie des États-Unis se raffermissait, celles d'Asie du Sud-Est enregistraient une croissance encore plus rapide. 
Sud-Est, malgré les conséquences néfastes manifestes du rattachement sur la compétitivité-coûts au niveau international. Au contraire, après avoir à peu près arrimé leur monnaie au dollar américain depuis l'établissement des taux de change flexibles, à partir du début des années 90 et surtout du milieu de la décennie, la plupart des banques centrales d'Asie du Sud-Est ont résisté aux ajustements à la baisse de leur taux de change, ce qui aurait réduit, voire évité, certains des effets déstabilisateurs des récentes débâcles monétaires. ${ }^{8}$

Selon la Banque des règlements internationaux (BIRD) (Asian Wall Street Journal, 6 janvier 1998), les emprunts internationaux auprès des banques commerciales étaient pour plus de la moitié à court terme, c'està-dire assortis de brèves échéances : Malaisie 56 pour cent, Thaïlande 66 pour cent, Indonésie 59 pour cent et Coré du Sud 68 pour cent. De plus en plus de données montrent que les banques européennes ont continué de prêter à des clients est-asiatiques, en dépit des mises en garde de la BIRD et d'autres institutions, bien avant que la crise n'éclate en juillet 1997 (Raghavan 1998).

Contrairement aux prétentions selon lesquelles «le marché» imposera rapidement des sanctions douloureuses aux gouvernements et aux économies qui n'ont pas procédé à une restructuration macroéconomique, le moment, la nature et les conséquences de la crise financière qui a frappé l'Asie du Sud-Est en 1997 font ressortir le caractère imparfait des marchés de capitaux. Ce dernier s'est manifesté à travers le long retard de la «rectification». Par exemple, les déficits des transactions courantes étaient plus graves en 1995 qu'en 1997, mais il n'y a eu alors aucune rectification, encore moins de punition des coupables, puisque le déficit courant en Malaisie et

${ }^{8}$ Au milieu des années 90, alors que le dollar américain se raffermissait avec l'économie américaine, les Japonais et les Allemands ont laissé leur monnaie se déprécier par rapport au dollar américain afin de restaurer leur compétitivité internationale, ce qui n'a provoqué que des perturbations assez faibles. 
dans certains autres pays voisins avait atteint des sommets inégalés, sans effet préjudiciable proportionnel sur ces économies. ${ }^{9}$

Dans le sillage de la crise mexicaine, au début de 1995, même le Fonds monétaire international (FMI) a momentanément renoncé à promouvoir une libéralisation financière presque débridée. L'horizon à court terme des marchés financiers vaut hélas aussi pour les mémoires humaines et institutionnelles et pour la politique et les recommandations économiques. La crise récente a en outre donné à voir un marché où l'ampleur du «surajustement» dépasse plusieurs fois celle de la «correction». On peut constater d'autres signes d'anarchie causée par les marchés dans le «comportement moutonnier» à l'origine des effets de «contagion» ou de «domino». Alors que les gouvernements et les économies ont été durement touchés par la crise depuis le milieu de 1997, peu de faits indiquent que les coupables du secteur privé en aient le plus souffert, c'est-à-dire que non seulement le marché n'est ni efficace ni rapide, mais qu'il est aussi injuste.

${ }^{9}$ Fait révélateur, au début de septembre 1997, Stanley Fisher, directeur adjoint du FMI, a observé que bien que les déficits des transactions courantes en Asie dn SudEst soient apparus plusieurs années auparavant, l'ajustement des marchés ne s'était pas produit-contrairement aux prévisions de la théorie économique classique. (Au lieu de reconnaître l'échec des mécanismes du marché, Alan Greenspan, président de la Réserve fédérale des États-Unis, réprimanda doucement Fisher en retour, comme s'il attendait du FMI qu'il «rappelle» à Wall Street ce que cette Bourse avait oublié). Pendant ce temps, «le marché» est devenu si obnubilé par le déficit des transactions courantes que cet indicateur, presque à lui seul, est devenu le fétiche des analystes financiers, surtout depuis la débâcle mexicaine du début de 1995. Auparavant, à des époques différentes, certaines économies ont maintenu beaucoup plus longtemps des déficits semblables, sans subir de conséquences comparables. Comme on l'a noté immédiatement après la crise mexicaine de 1995, plusieurs économies du Sud-est asiatique accusaient alors déjà des déficits comparables aux transactions courantes, en dépit de la croissance économique rapide, ou plutôt à cause d'elle. Cependant, comme l'a observé Fisher, les marchés monétaires n'ont pas réussi à s'ajuster plus tôt en Asie du Sud-Est. 
Du fait que la région de l'Asie du Sud-Est était perçue comme beaucoup plus intégrée qu'elle ne l'est en réalité (en termes de liens commerciaux, à l'exception de Singapour, l'entrepôt régional), les décisions d'investissement prises sous l'effet de la panique par les gestionnaires de fonds hors de la région (à Wall Street ou à Londres) ont souvent été «moutonnières» ${ }^{10}$ ce qui a provoqué un effet de «contagion» ou de «domino» dans toute la région. La logique et l'ampleur même des opérations des fonds de couverture ${ }^{11}$ ont cu tendance à exacerber ces phénomènes, ce qui a entraîné un effet boule de neige catastrophique pour la région. Les autres spéculateurs et opérateurs en couverture internationaux, régionaux et, de plus en plus, locaux, ont aussi leur part de responsabilité, mais ont surtout réagi par intérêt personnel aux tendances du marché qu'ils percevaient, plutôt que dans le cadre de quelque vaste conspiration.

\section{Leçons de politique monétaire}

À quoi bon affirmer que la crise n'aurait pas dû se produire puisque les fondamentaux économiques est-asiatiques étaient solides, même si cela était vrai ? Dans certains cas, cette dénégation a exacerbé la crise, car les autorités ne l'ont pas reconnue et n'y ont pas remédié avec un grand sentiment d'urgence. Malheureusement, comme l'a appris l'Asie de l'Est à ses dépens, les marchés de capitaux sont guidés par les sentiments autant que par les fondamentaux. Par conséquent, bien qu'en 1995 des déficits courants beaucoup plus graves n'aient pas entraîné de crise, cela ne saurait signifier qu'une

${ }^{10}$ En présence d'informations limitées et d'une situation nouvelle qui évolue rapidement, un tel comportement, quoique regrettable, est souvent considéré comme rationnel par les opérateurs du marché

"I Les fonds de couverture peuvent toutefois adopter des orientations différentes, par exemple lorsque le déscngagement sur une monnaie opéré par un fonds en amène un autre à acquérir des actifs à vil prix : faisant preuve de persévérance, les étrangers ont souvent été des acquéreurs nets d'actions nippones, pendant toute la période des années 90 où la bulle a éclaté au Japon. 


\section{Africa Development, Vol. XXV, Nos. 1 \& 2, 2000}

économie puisse indéfiniment maintenir de tels déficits sans être exposée à une attaque spéculative ou à une perte de confiance.

À titre d'exemple, on ne peut libéraliser les mouvernents de capitaux, puis se plaindre lorsque des opérateurs fantasques retirent brusquement leurs investissements de portefeuille à court terme. Même le Chili, autrefois l'enfant chéri des monétaristes de Chicago, rend très difficile et coûteux le retrait rapide de capitaux de son économie et traite très différemment les investissements directs étrangers des investissements de portefeuille. Dans ce cas,les autorités tentent de distinguer entre les investissements de portefeuille qui sont simplement à court terme et, disons; les fonds de pension davantage orientés à moyen terme. Après tout, on ne peut s'attendre à ce que le nombre d'oiseaux qui entrent à tire-d'aile dans une cage ouverte dépasse indéfiniment le nombre de ceux qui en sortent, puisque la prémisse fondamentale de la libéralisation financière est «un clou chasse l'autre». ${ }^{12}$

Au cours des dernières années, certaines économies d'Asie du Sud-Est sont devenues beaucoup trop tributaires de tels apports de capitaux à court terme pour combler leur déficit des transactions courantes. Ce problème a été exacerbé par des importations excessives destinées à produire davantage de biens non exportables, notamment des bâtiments et des infrastructures. Au lieu de consentir des crédits à des fins plus productives, les institutions financières qui affichent la prudence préfèrent souvent accorder des prêts en vue de financer des acquisitions de biens immobiliers ou d'actions, et ainsi obtenir en garantie des actifs dont la valeur croît.

Alors que les banques étrangères étaient bien aises de prêter des dollars américains à des taux d'intérêt plus élevés qu'ailleurs, les entreprises d'Asie du Sud-Est étaient ravies d'emprunter à des taux

${ }^{12}$ La libéralisation financière signifie que les investisseurs ont le choix d'aller et venir et, bien entendu, l'existence même de ce choix peut les encourager à rester dans certaines circonstances. 
inférieurs à ceux du marché intérieur. Rétrospectivement, le coût de la couverture-environ cent points de base pour le ringitt-dollar, quelques centaines pour le baht-dollar ou la roupie-dollar- parait maintenant faible. L'existence d'un marché bien développé de swaps permet aux entreprises d'Asie du Sud-Est de recourir aux marchés de capitaux étrangers, à un coût raisonnable, en éliminant le risque de change par des opérations de swaps. Le problème a donc été en fin de compte celui de l'avidité : les taux d'intérêt beaucoup plus bas à l'étranger conjugués à des taux de change apparemment fixes ont amené les emprunteurs à se lancer dans des opérations hasardeuses, sans payer le coût d'une certaine assurance en se prémunissant contre les risques.

Par conséquent, la plupart de ces prêts sont restés non couverts, tandis que les monnaies du Sud-Est asiatique semblaient arrimées au dollar américain depuis les années 70 , même si officiellement, les taux de change étaient censés varier en fonction d'un panier de monnaies des principaux partenaires commerciaux étrangers. L'essor du secteur bancaire privé observé ces dernières années dans la région a débouché sur une surenchère des prêts, non sans rappeler les crédits accordés aux États du tiers-monde à la fin des années 70 (dont l'accumulation a conduit à la crise de la dette du début des années 80). Cependant, parmi les décideurs internationaux, on se berçait désormais dans l'illusion qu'une telle accumulation de dettes du secteur privé était sans importance du moment que la dette publique était maîtrisée.

Pendant ce temps, les investissements de portefeuille se sont portés sur les nouvelles bourses émergentes d'Asie du Sud-Est, encouragés par la Société financière internationale, un organisme de la Banque mondiale. En Malaisie, par exemple, ils sont arrivés en grand nombre en 1993, si ce n'est qu'ils se sont retirés encore plus brusquement en 1994, laissant la plupart des petits porteurs en mauvaise posture. Néanmoins, les responsables de la conduite des affaires publiques semblent hélas avoir la mémoire courte et n'avaient 


\section{4}

Africa Development, Vol. XXV, Nos. $1 \& 2,2000$

pas tiré les leçons de l'expérience lorsqu'à partir de 1995, par suite de la nouvelle accumulation insoutenable, les cours des actions se sont à nouveau envolés, en dépit de la décrue des coefficients de capitalisation des résultats. Le reste c'est de l'histoire, mais comme l'a dit un sage, lorsque l'histoire se répète, la première fois, c'est une tragédie et la deuxième, une farce.

Ainsi, les crises monétaire et financière survenues en Asie du SudEst depuis le milieu de 1997 ont été dues en partie à la libéralisation financière et au fait que la gestion monétaire et financière s'en est trouvée compromise. Le «quasi-rattachement» des monnaies de la région au dollar américain et l'encouragement des apports de capitaux étrangers-qui se sont tournés vers les bourses récemment ouvertes ou ont pris la forme d'emprunts, souvent à court terme ${ }^{13}$ _destinés à combler le déficit courant, ont aussi permis à l'épargne étrangère de compléter le taux d'épargne intérieur déjà élevé, pour relever les taux d'investissement dans la région, ce qui a contribué à une forte augmentation de la bulle des prix des actions et de l'immobilier. Le quasi-rattachement a non seulement encouragé les emprunts non garantis à l'étranger, mais est aưssi devenu la cible des spéculateurs monétaires, à mesure que les monnaies régionales s'appréciaient parallèlement au dollar américain, en dépit de la baisse de la compétitivité des exportations et de la croissance.

Dans le même temps, la libéralisation financière a offert des occasions lucratives de profiter pleinement de la chute des monnaies, ce qui a accéléré et exacerbé l'écroulement des marchés régionaux des changes et des actions. Outre la réaction inadéquate des pouvoirs publics, tout cela a transformé l'inévitable «correction» des monnaies surévaluées de la région en un effondrement des monnaies et des marchés boursiers de la région, en proie à une panique aggravée par le comportement «moutonnier» et la «contagion».

${ }^{13}$ Les investissements à court terme, encouragés par la libéxalisation financière, ont aussi accentué le parti pris contre les investissements productifs à plus long terme. 
Bien que les systèmes financiers de la région soient assez variés et loin d'être des copies conformes du système japonais doté de plusieurs grandes banques (comme on l'a souvent affirmé à tort), du fait de telle ou telle conjoncture, ils se sont néanmoins trouvés exposés au même phénomène de «bulle» financière et immobilière, quoique pour des raisons quelque peu différentes. On peut dire que dans les systèmes de la Thaillande, de la Corée et de l'Indonésie, où les banques prédominaient, il existait un lien de ce type plus fort qu'en Malaisie, où le système financier était beaucoup plus orienté vers le marché boursier. La croissance rapide, reposant sur 1'industrialisation tournée vers les exportations à partir de la fín des années 80, a donné naissance à une expansion financière non réglementée, qui a contribué à un essor de l'immobilier et à la formation de bulles des prix des actifs, à la fois en Malaisie, à l'économie de marché plus «anglo-saxonne», et en Thaillande, accordant une plus large place aux banques.

Par suite de l'effondrement des monnaies, les actifs que les investisseurs à court terme avaient acquis dans la région (investissements de portefeuille et autres) se sont dévalorisés en conséquence, d'où un désengagement et une panique encore pires qui ont provoqué un mimétisme et sans doute une contagion qui s'est étendue, par-delà les frontières nationales, au reste de la région. En Malaisie et sans doute ailleurs, d'autres effondrements des marchés immobiliers et boursiers paraissent imminents, étant donné les constructions excessives dépourvues de coordination et le lien immobilier-finances. Par conséquent, de nombreuses économies ont été heurtées par ce triple choc des marchés des changes, des actions et de l'immobilier, ou risquent encore de l'être.

La hausse des taux d'intérêt exigés par les milieux financiers en 1998 a retourné le couteau dans la plaie, mais sans guère réussir à attirer de nouveau les capitaux à court terme. Mais même lorsqu'ils reviennent, de tels apports ne peuvent être que temporairement maintenus et conservés, moyennant un coût élevé et permanent pour 
les investissements productifs dans l'économie réelle. Et si ces flux finissent par être inversés avec la précipitation qu'a connue l'Asie du Sud-Est au second semestre de 1997, beaucoup de répercussions annexes seront à nouveau subies.

Du fait de ces bouleversements, l'Asie du Sud-Est a été appelée à opérer des réformes de politique intérieure possédant quatre aspects, à savoir la flexibilité accrue des taux de change, l'urgence de la réforme du secteur financier, les bulles des prix des actifs et le déficit courant. Avant d'analyser les défis au niveau national et international, il convient de résumer les quatre dimensions de la crise actuelle.

À moins que les économies avancées ne stabilisent les taux de change entre leurs monnaies respectives, le quasi-rattachement du taux de change d'une économie était devenu très dangereux, ainsi que l'avait démontré la crise récente. Les apports de capitaux à court terme peuvent temporairement compléter l'épargne intérieure, mais leur reflux risque de provoquer de graves perturbations. Bien que ces flux puissent être influencés par les fondamentaux économiques à long terme, ils obéissent en général à des considérations spéculatives à brève échéance. Des ajustements du taux de change à court terme, qui entraînent des effets déstabilisateurs sur les prix intérieurs et les salaires, sont alors jugés nécessaires pour endiguer les brusques sorties de capitaux, mais ils offrent par ailleurs des occasions aux spéculateurs monétaires.

La réforme du secteur financier doit être envisagée, non seulement sous l'angle de la libéralisation réclamée par les intérêts financiers internationaux, mais aussi sur le plan de la nouvelle réglementation prudentielle indispensable pour prévoir et résoudre les nouvelles difficultés. Bien que les tenants de la libéralisation insistent souvent sur les problèmes causés par une réglementation excessive et inopportune, une politique bancaire libérale peut se traduire par un 
secteur bancaire intérieur faible,,$^{14}$ incapable de résister à la concurrence étrangère, et même par l'effondrement ou le sauvetage coûteux d'établissements affaiblis. Pour la plupart des économies en développement, des politiques de «rigueur financière» restent par ailleurs nécessaires pour «diriger» le crédit ${ }^{15}$ afin de financer des investissements productifs, surtout dans les domaines prioritaires, plutôt que l'acquisition d'actifs ou la consommation. Les tendances récentes qui révèlent une convertibilité accrue aux fins des opérations en capital, des instruments financiers novateurs, ainsi que la multiplication des centres financiers extraterritoriaux, des sociétés financières non bancaires et des «activités bancaires privées» posent aussi de nouveaux problèmes aux autorités chargées de la réglementation financière, compte tenu de la transparence réduite.

Le crédit facile, en partie dû aux entrées de capitaux, a entraîné une montée vertigineuse des prix de l'immobilier ainsi que des cours des actions, souhaitée par la plupart des intéressés. Une réglementation bancaire visant à réduire autant que possible une telle hausse des prix des actifs mérite de recevoir la priorité absolue et est toujours difficile à mettre en place, lorsque tout va bien, sans provoquer un effondrement des prix des actifs, mais elle a été plus facile à établir après l'éclatement de la bulle des prix des actifs.

Comme dans beaucoup d'autres situations de croissance rapide, pendant les années 90 , on en est venu à considérer les déficits courants comme «naturels» en Asie du Sud-Est, avant la crise, car on estimait qu'ils ne faisaient que refléter l'excès des investissements

${ }^{14}$ Comme au Chili, au début des années 80 .

${ }^{25}$ À ce titre, la politique fiscale peut encourager les épargnants en s'abstenant de les pénaliser lorsqu'ils amassent des économies. Même s'il convient que les banques prennent toujours des décisions de prêt fondées uniquement sur des critères économiques, il faut atténuer les préjugés systémiques en faveur des opérations à court terme. Les autorités peuvent privilégier certains types d'investissement en les subventionnant à travers la fiscalité ou en accordant des garanties de prêt aux secteurs ou activités qu'elles jugent importants. 
dans l'économie nationale par rapport à l'épargne intérieure; de ce fait, certains responsables ne s'en étaient pas réellement préoccupés. Depuis la crise de la dette du début et du milieu des années 80 , la réduction des déficits budgétaires a reçu la priorité absolue, sur l'ordre des institutions de Bretton Woods et autres. L'évolution depuis la crise mexicaine du début de 1995 semble indiquer que le déficit courant était le talon d'Achille des économies d'Asie du Sud-Est, précipitant au bout du compte les crises financières qui ont commencé par la chute des monnaies de ces pays (quasiment rattachées au dollar américain), ce qui a, par inadvertance, encouragé des emprunts privés massifs, souvent non couverts, auprès de l'étranger.

\section{L'évolution du système financier international}

Les critiques ${ }^{16}$ de Mahatir Mohamad, le premier ministre de la Malaisie, à l'encontre du rôle joué par la spéculation monétaire internationale, qui aurait précipité la récente crise est-asiatique, et des réactions du FMI, ont été largement rejetées en dehors de la Malaisie, sauf par ceux qui reconnaissent que ces observations traduisent une frustration troublée, face à un nouveau phénomène auquel l'analyse économique classique n'apporte pas d'explication satisfaisante. Rejeter les arguments de Mahatir équivaudrait à jeter le bébé avec

${ }^{16}$ Il a été très difficile à la Malaisie de se poser de façon plausible en grand défenseur de la morale à propos de la spéculation monétaire et autres, en raison du comportement bien connu de la banque Negara, pendant les années 80 . Il était connu que la banque centrale malaisienne prenait des positions à court terme très risquées sur les principales devises, en vue d'en tirer profit. Cette situation s'est poursuivie pendant plusieurs années, jusqu'à ce que la banque perde plusieurs dizaines de milliards de ringgit en 1992, alors qu'elle pariait sur la livre, et opte ensuite pour des activités plus paisibles. On estime donc à l'étranger que les commentaires de Mahatir sont de mauvaise foi, car par le passé, il a chargé son gouvernement de se lancer dans des activités spéculatives et a été en mesure de le faire grâce à la très grande ouverture des marchés internationaux des changes et des produits de base. Après avoir approuvé de telles activités, il a été difficile d'attirer une compassion liée aux agissements des spéculateurs non malaisiens. 
l'eau du bain, car le Premier ministre tentait d'aborder un problème réel, même s'il s'y prenait mal. Après tout, comme beaucoup l'ont déjà signalé, le système financier international et la poursuite de sa libéralisation ont favorisé ceux qui jouissaient déjà d'une position dominante et privilégiée dans l'économie mondiale, aux dépens de l'économie réelle et du «développement tardif» dans le Sud.

Ironie du sort, l'ennemi juré de Mahatir, le financier international George Soros, a fait valoir que l'expansion non réglementée du capitalisme, en particulier du capitalisme financier, menace de compromettre l'avenir de ce système, c'est-à-dire qu'il faut sauver le capitalisme de lui-même. Tout en admettant qu'il a pour sa part largement profité de la libéralisation financière, Soros a affirmé, avec des accents keynésiens, que la libéralisation excessive a abouti à une quasi-anarchie, qui met en péril la stabilité si indispensable à la croissance capitaliste ordonnée et au développement démocratique souhaités dans sa vision libérale d'une «société ouverte» prônée par Popper.

L'actuel système de taux de change flexibles a été instauré il y a plus d'un quart de siècle, inaugurant un nouveau système monétaire international aux effets très contrastés. Par conséquent, ce dernier est assez récent, puisqu'il n'a débuté qu'après que Richard Nixon, président des États-Unis, ait décidé en 1971 le retrait unilatéral du système de taux de change fixes de Bretton Woods, dans lequel le dollar américain était rattaché à l'or, à raison de 35 dollars américains l'once, et le ringitt arrimé au dollar américain, au taux de 3 ringitt pour un dollar américain. Dans le cadre du nouveau système, en 1995, le volume d'opérations de change au comptant avait atteint plus de 67 fois la valeur totale du commerce international des biens, soit plus de 40 fois la valeur du total des échanges internationaux (y compris les «invisibles» ou les services). ${ }^{17}$ Dans une perspective historique, ces

${ }^{17}$ Puisque les opérations de change liées au commerce sont largement inférieure à celles qui se rapportent aux «investissements», il n'est pas étonnant que le volume 


\section{Africa Development, Vol. XXV, Nos. $1 \& 2,2000$}

opérations de change ne sont donc guère naturelles, inévitables, ni même souhaitables. Pendant la plus grande part de l'histoire de l'humanité, y compris celle du capitalisme, elles n'ont pas fait «partie intégrante du commerce mondial des biens et des services», comme l'a prétendu à l'époque le ministre de l'économie et des finances des États-Unis, Robert Rubin. En réalité, comme il est bien connu, divers critiques ont proposé différentes formules de rechange au système actuel, dont le retour aux taux de change fixes, l'étalon-or, etc.

Dans une économie mondiale où les opérations de change au comptant représentent maintenant plus de soixante-dix fois la valeur des transactions internationales au titre du commerce des produits de base, le secteur financier est de plus en plus coupé de l'économie réelle. Compte tenu de la récente multiplication de nouveaux instruments et marchés financiers, notamment en Malaisie, le secteur financier risque encore plus d'avoir des effets préjudiciables sur l'économie réelle. Depuis que Lord Keynes a recommandé de «jeter du sable» dans le système financier pour vérifier les conséquences désastreuses de la libéralisation débridée, les keynésiens-et d'autres-se sont méfiés de la libéralisation financière préconisée par les néolibéraux au nom de leur idéologie et par leurs alliés souvent naïfs.

Quoique l'on prétende que «le marché» imposera rapidement de douloureuses sanctions aux gouvernements et aux économies dont la situation macroéconomique n'est pas saine, le moment, la nature et les répercussions de la crise financière qui a frappé l'Asie du Sud-Est au milieu de 1997 soulignent le caractère imparfait des marchés financiers. Ce dernier s'est manifesté à travers le long retard de la

des opérations de change soit si important. L'une des questions essentielles consiste à déterminer combien de ces échanges liés aux investissements sont «sains», «opportuns» ou «souhaitables», ce qui est difficile à dire. Les investisseurs internationaux veulent protéger leur revenu et leur patrimoine personnels en répartissant leurs investissements entre de nombreux pays et en les modifiant très souvent, à mesure que les conditions évoluent, ce qui contribue à l'instabilité des marchés. 
«rectification». Fait révélateur, en septembre 1997, Stanley Fisher, directeur adjoint du FMI, a signalé que même si l'apparition de déficits courants en Asie du Sud-Est remontait à quelques années auparavant, les marchés ne s'étaient pas ajustés, contrairement aux prédictions de la théorie économique classique. (En guise de réponse, au lieu de reconnaître l'échec des mécanismes du marché, Alan Greespan, président de la Réserve fédérale des États-Unis, a réprimandé Fisher, comme s'il attendait du FMI qu'il «informe» Wall Street de cette omission.)

L'instabilité du marché se traduit aussi par l'ampleur du «surajustement» des taux de change, qui semble avoir été plusieurs fois supérieure à l'inévitable «correction». On peut constater d'autres signes de l'anarchie provoquée par le marché dans le «comportement moutonnier» à l'origine des effets de «contagion» ou de «domino». Après la crise mexicaine, au début de 1995, le Fonds monétaire international (FMI) s'est temporairement abstenu de promouvoir une libéralisation financière presque sans retenue. Pendant ce temps, les déficits courants avaient atteint des sommets inégalés dans certaines économies d'Asie du Sud-Est, sans effets néfastes comparables. Malheureusement, l'horizon à court terme des marchés financiers vaut aussi pour nos souvenirs ainsi que pour la formulation de la politique et des recommandations économiques.

James Tobin, lauréat du prix Nobel de sciences économiques, a réclamé la création d'une taxe sur les opérations de change au comptant, pour permettre la conduite d'une politique monétaire nationale plus indépendante, décourager les mouvements de capitaux spéculatifs et accroître le poids relatif des données économiques fondamentales à long terme, par rapport aux considérations spéculatives et à courte vue. De surcroît, la taxe perçue aurait l'avantage de produire un financement plus que suffisant pour le système et les programmes des Nations Unies, au lieu de le laisser à la merci des caprices des dirigeants américains, comme cela se produit depuis longtemps. Un autre lauréat du prix Nobel, Lawrence 
Klein, a cité deux autres formules à envisager, outre la taxe Tobin, à savoir des mécanismes monétaires régionaux ainsi que l'intégration de «coupe-circuits» dans le système, suggestion également formulée par Joseph Stiglitz, premier vice-président et économiste en chef de la Banque mondiale.

Cependant, le lobby en faveur de la libéralisation financière reste bien plus puissant et beaucoup plus influent, puisqu'il domine la plupart des journaux d'affaires et les principales institutions financières au niveau international, surtout aux États-Unis. Admettant que l'argent est un produit doté d'une spécificité particulière, le Wall Street Journal, par exemple, continue de promouvoir les caisses d'émission (au lieu des banques centrales) et l'arrimage d'autres monnaies au dollar américain, tout en attaquant la plupart des autres régimes monétaires internationaux et en concédant rarement les avantages que le rattachement au dollar procure aux États-Unis, par exemple le fait de faire financer leurs énormes déficits par le reste du monde.

\section{Conséquences de la libéralisation financière}

Une explosion des flux financiers internationaux a suivi le remplacement du système de taux de change fixes de Bretton Woods par un nouveau système de taux de change flexibles. On attribue en général de puissants motifs spéculatifs aux flux internationaux de capitaux. Toutefois, la détente des taux de change fixes est aussi allée de pair avec un assouplissement des contrôles de capitaux, ce qui a permis à de nombreux investisseurs de diversifier leurs portefeuilles avec profit. En tout cas, cette tendance s'est accélérée à partir des années 80, ce qui a débouché, en 1997, sur un marché des changes aux transactions quotidiennes de 1250 milliards de dollars américains et sur la prolifération de nouveaux instruments financiers. Toutefois, un grand nombre des prétendus bienfaits de la libéralisation financière n'ont pas été récoltés, comme le montre le résumé suivant des constatations récentes d'Eatwell (1997). 
- Premièrement, on s'attendait que la libéralisation financière permette de transférer des ressources des pays riches aux pays pauvres en capitaux ${ }^{18}$ - alors qu'en fait, les flux nets de financement--et de ressources réelles-ont été très modestes et principalement orientés vers les pays riches en capitaux. ${ }^{19}$ Bien entendu, la plupart des flux nets vers les pays «pauvres en capitaux» se sont surtout dirigés vers les «marchés émergents» comme ceux d'Asie de l'Est, ce qui, peut-on dire, a contribué à la formation de bulles des actifs et, en fin de compte, à la panique financière et à l'effondrement des marchés monétaires et boursiers.

${ }^{18}$ De rêcents résultats démontrent que l'épargne nationale tend à égaler l'investisse-ment national, ce qui porte à croire que les flux de capitaux se dirigeant vers «l'utilisation optimale» sont loin d'être universels et beaucoup plus faibles que ne le prédisent certaines théories simplistes. Le manque d'information ou d'autres risques et incertitudes ont tendance à réduire les mouvements de capitaux internationaux.

${ }^{19}$ Eatwell semble indiquer une corrélation négative entre la dépendance à l'égard de l'«épargne étrangère» et les résultats économiques. Cela est vrai si l'épargne étrangère n'est pas ventilée en fonction de sa nature. Les chiffres sont fortement biaisés par l'inclusion des flux à court terme du marché monétaire, qui peuvent tenir aux efforts des pouvoirs publics de soutenir la monnaie nationale en pratiquant des taux d'intérêt élevés qui attirent temporairement des capitaux étrangers. Le Mexique, le Brésil et surtout le Venezuela, en ont êté des exemples typiques, il y a quelques années. Si l'on ne tenait compte que des investissements directs ou des prises de participation à long terme, un grand nombre d'économies latino-américaines aux résultats médiocres n'en feraient pas partie. Les pays d'Asie du Sud-Est, en particulier Singapour et la Malaisie, auraient alors un classement élevé, en fonction de l'épargne étrangère (évaluée «convenablement») et des résultats économiques. 
épargnants qui ont le plus tiré parti de la hausse des taux d'intérêt réels.20

- Troisièmement, les nouveaux produits dérivés financiers-qui devaient améliorer la gestion des risques - ont en fait engendré de nouveaux risques systémiques et sont particulièrement vulnérables à de brusques revirements d'opinion. ${ }^{21}$

${ }^{20}$ Actuellement, les taux d'intérêt élevés sont un fait très regrettable pour la région. Ils visent en partie à soutenir la monnaie en vae de maintenir la confiance, mais surtout peut-être, de permettre aux entreprises locales de rembourser leurs dettes vis-à-vis de l'étringer. Le prix de cette situation est un ralentissement de la croissance. Avec des taux d'intérêt et des taux de change plus bas, qui favoriseraient la croissance de l'économie et aideraient les consommateurs, les entreprises locales mal gérées devraient se réorganiser, ou alors perdre leurs actifs (ce qu'elles méritent dans certains cas). Les créanciers étrangers qui ont été assez sots pour prêter des dollars à des entreprises mal gérées devraient voir leurs prêts et leurs obligations bancaires faire l'objet d'une défaillance. Les entreprises locales en faillite pourraient être renflouées et recapitalisées, la totalité des parts du capital social étant versée dans des fonds communs de placement ou des fonds de pension et distribuée de façon égale aux masses populaires. La libéralișation va en général de pair avec des taux d'intérêt élevés. Cependant, des taux d'intérêt plus bas auraient pu résulter d'une conjonction de taux de change fixes, de contrôles des capitaux et du déploiement de capitaux au sein de ces économies. Les taux de change fixes sont maintenus par des contrôles de capitaux qui «emprisonnent» les ressources d'épargne dans une économie. Celles-ci sont en général exploitées par les gouvernements ou les cartels bancaires, qui peuvent maintenir les taux d'intérêt à des niveaux trop bas, inférieurs même au taux d'inflation. Les contrôles de capitaux risquent donc d'obliger les épargnants à accepter des taux d'intérêt faibles et de les empêcher d'obtenir ailleurs un rendement plus équitable. Les ressources d'épargne obtenues à bon marché peuvent être prêtées à des sociétés peu méritantes, ou servir à d'autres fins, peut-être selon les directives des powvoirs publics.

${ }^{21}$ On pourrait affirmer qu'une partie de cette situation résulte de l'avidité, de la stupidité et du manque de connaissances ou de réglementation. S'ils sont utilisés avec prudence, les produits dérivés sont en fin de compte des contrats d'assurance. 
- Quatrièmement, l'amélioration de la performance macroéconomique-comportant une hausse de la croissance et de l'investissement escomptée d'une meilleure efficacité de la répartition-ne s'est pas produite; en fait, la performance macroéconomique globale a été pire qu'avant la libéralisation. ${ }^{22}$

- Cinquièmement, au lieu d'imposer aux autorités une «saine discipline» devant améliorer la stabilité macroéconomique, la libéralisation financière a introduit une orientation déflationniste persistante dans la politique économique, alors que les pouvoirs publics tentent d'acquérir une crédibilité pour éviter de déstabiliser les mouvements de capitaux.

Les marchés financiers paraissent opérer de manière à imposer leurs propres «anticipations» à l'économie réelle, définissant ainsi leur propre logique et leurs propres «fondamentaux», ces prédictions pouvant à leur tour finir par se réaliser d'elles-mêmes. Autrement dit, les marchés ne se bornent pas à traiter l'information en vue de répartir efficacement les ressources. Puisqu'ils fonctionnent comme des concours de beauté et que l'économie réelle n'a pas automatiquement tendance à converger vers la croissance du plein-emploi, les hypothèques analytiques présumées d'autres opérateurs des marchés sont imposées à l'économie.

La menace d'instabilité sur les marchés financiers maintenant énormes oblige les pouvoirs publics et les investisseurs privés à adopter des stratégies de prévention des risques, qui se soldent par une faiblesse de la croissance et de la création d'emplois. Face au risque onéreux d'enfreindre les règles du jeu, il apparaît une orientation déflationniste dans la politique des pouvoirs publics et dans le secteur privé. Cette distorsion est exacerbée par le coût élevé

${ }^{23}$ Du fait de la séparation entre la détention et la gestion des investissements de portefeuille, bien qu'il puisse être dans l'intérêt des investisseurs «d'acquérir et de conserver», il est difficile de rédiger des contrats qui encouragent les gestionnaires de fonds de pension ou de fonds communs de placement et d'autres intermédiaires à ne pas céder leurs actifs. 
de l'endettement, imputable aux taux d'intérêt réels élevés, destinés à maintenir la stabilité financière dans un monde potentiellement instable. Par conséquent, la «stabilité des prix à long terme» remplace un niveau élevé et stable d'emploi comme priorité d'action des pouvoirs publics. Un tel système stable sur le plan monétaire, assorti d'une croissance assez lente et d'un chômage élevé, peut durer indéfiniment.

Un système financier complexe et libéralisé, accordant la priorité à la flexibilité ou à la possibilité d'une sortie facile du marché, est nécessairement fragile, comme le montrent :

- les crises de liquidité, qui réduisent la production réelle ;

- la frilosité du secteur privé, qui encourage une vision à court terme $^{23}$;

- le souhait du secteur public d'éviter les risques, qui se traduit par une orientation déflationniste de l'action des pouvoirs publics ;

- des pressions persistantes en vue d'une flexibilité accrue, facilitant la sortie du marché.

Il convient de mettre en balance les avantages qu'a apportés la réduction des contrôles financiers aux «marchés émergents» et l'instabilité accrue, imputable à la facilité renforcée de sortie. Alors que la hausse des investissements directs étrangers (réels) nécessite en général un accord sur le rapatriement sans restrictions des

${ }^{23}$ Du fait de la séparation entre la détention et la gestion des investissements de portefeuille, bien qu'il puisse être dans l'intérêt des investisseurs «d'acquérir et de conserver», il est difficile de rédiger des contrats qui encouragent les gestionnaires de fonds de pension ou de fonds communs de placement et d'autres intermédiaires à ne pas céder leurs actifs. 
bénéfices, ces conditions sont bien différentes de la «sortie instantanée» exigée par les marchés de capitaux. ${ }^{24}$

De nombreux faits montrent qu'à long terme, le développement économique a été lié à des stades. L'âge d'or de l'après-guerre, qui a coïncidé avec des niveaux élevés de production et d'emploi et une efficience de courte durée, reposait sur une gestion macroéconomique active dans le cadre du système de Bretton Woods. La reconstruction européenne de l'après-guerre a été réalisée grâce à de rigoureux contrôles des capitaux. En revanche, suite à la récente ruée vers la convertibilité et la déréglementation des mouvements de capitaux en Europe de l'Est, la Russie est devenue un grand exportateur net de capitaux. $^{25}$

Certains dangers liés à la libéralisation financière sont maintenant devenus assez évidents, mais la plupart ne sont pas suffisamment reconnus, et encore moins débattus, ni abordés. La majorité des initiatives dans ce domaine ne peuvent être engagées à titre unilatéral sans un coût élevé, comme l'ont clairement indiqué les réactions aux critiques formulées par $\mathrm{M}$. Mahatir, le Premier Ministre de la Malaisie. Il y a lieu d'examiner avec soin les possibilités très restreintes d'initiatives unilatérales et de ne les concrétiser que si elles sont jugées souhaitables. Le fait de citer de manière sélective des cas dans lesquels l'élaboration ou l'application de la politique des pouvoirs publics ont été erronées et caractérisées par l'incompétence ne saurait justifier que l'on s'en remette à des marchés libéralisés qui rendent impossible la définition systématique d'une ligne de conduite. $\mathrm{Au}$ contraire, cette situation souligne l'importance de créer les

${ }^{24}$ Bien entendu, la liquidité est l'une des caractéristiques qui amène des investisseurs autrement frileux à $s^{3}$ engager dans une situation. En outre, dans toute transaction, 1 existe un acheteur pour tout vendeur.

Naturellement, l'exode des capitaux n'est pas la conséquence inévitable de la libéralisation financière, mais peut correspondre à des craintes et partant, à des opérations de couverture réalisées par les négociateurs individuels de parquet. 
conditions et de se doter de la capacité permettant à une bonne politique adaptée aux circonstances d'être efficace.

Il importe de rechercher activement à mettre en place un grand nombre de mesures par voie d'initiatives multilatérales, pour lesquelles les autorités ont besoin du soutien des États voisins et autres. Compte tenu de la puissance de l'idéologie dominante qui imprègne le système intemational actuel, il est quasiment impossible d'exercer un contrôle sur le système financier sans une modification fondamentale des priorités et de la réflexion des principaux gouvernements concernés. Néanmoins, les monnaies d'un petit nombre de ces pays-les États-Unis, l'Allemagne et le RoyaumeUni-intervenaient dans plus des trois quarts des opérations monétaires en 1995. Par conséquent, en agissant de concert, ces gouvernements ont la capacité de contrôler les flux de capitaux, mais bien sûr, seulement s'ils renient leur foi dans la prétendue supériorité du néolibéralisme.

\section{Une crise d'un nouveau genre}

On peut affirmer à juste titre que personne n'avait entièrement prévu la crise en Asie de l'Est. Bien sûr, certains sceptiques considéraient les proclamations d'un miracle est-asiatique comme un tant soit peu exagérées, quoique pour diverses raisons, notamment parce que ces économies n'avaient pas obtemu une grande croissance de la productivité et finiraient par se heurter à des rendements décroissants (Krugman 1994); d'autres soutenaient que les résultats des nouveaux pays industriels est-asiatiques étaient nettement inférieurs à ceux du Japon et des nouvelles économies industrielles de première génération (Jomo et al. 1997). 
Il apparaît maintenant clairement que la crise est-asiatique diffère des scénarios classiques de crise monétaire, au moins à plusieurs égards (Krugman 1998) ${ }^{20}$ :

1) l'absence des sources habituelles de tension monétaire, qu'il s'agisse de déficits budgétaires ou d'indiscipline macroéconomique ${ }^{27}$;

2) les pouvoirs publics n'avaient aucun intérêt à abandonner les taux de change fixes, par exemple pour réduire le chômage ;

3) les cycles marqués d'alternance d'essor et d'effondrement des prix des actifs (marchés immobiliers et boursiers) ont précédé la crise monétaire, surtout en Thaîlande, où la crise a débuté ;

${ }^{26}$ La tentative de Paul Krugman (1998) pour procéder à un rattrapage théorique mérite particulièrement d'être considérée à la lurnière de ses précédents efforts en vue de comprendre des phénomènes économiques internationaux reliés ainsi que la croissance économique est-asiatique. Alors que la crise continue d'évoluer, une telle recherche ne saurait être définitive, du fait, surtout, que nous ne pouvons même pas tirer pleinement parti de la rétrospection. Néanmoins, alors que la politique est tout à fait improvisée, il est fort probable que cette tentative pour faire ressortir certaines relations soit révélatrice. Krugman fait donc l'affirmation suivante : «Il convient d'adopter une approche tout à fait différente de celle de la théorie classique de la crise monétaire. Bien sûr, les économies asiatiques ont subi des crises monétaires et les canaux habituels de la spéculation ont été comme toujours utilisés. Cependant, ces dernières faisaient seulement partie d'une crise financière plus vaste, qui était sans grand rapport avec les monnaies ou mêrne les questions monétaires en tant que telles, ni avec les questions budgétaires traditionnelles. Au contraire, pour comprendre ce qui a déraillé, il faut nous attacher à deux sujets habituellement négligés par l'analyse des crises monétaires : le rôle des intermédiaires financiers (et le risque moral lié à de tels intermédiaires lorsque leurs activités étaient mal réglementées) et les prix des actifs réels, tels que les capitaux et les terrains»y.

27 Aucun des fondamentaux habituellement mis en évidence ne paraissent avoir été importants dans les économies touchées: tous les gouvernements enregistraient des excédents budgétaires et aucun n'était confronté à une expansion monétaire excessive, tandis que les taux d'inflation étaient généralernent faibles. 
4) le rôle déterminant joué par les intermédiaires financiers dans toutes les économies touchées;

5) la gravité de la crise en l'absence de rudes chocs néfastes ;

6) la rapide diffusion de la crise initiale survenue en Thaïlande, qui s'est même étendue aux économies ayant peu de liens ou de ressemblances avec les premiers pays victimes.

Il convient donc de noter que les indices traditionnels de vulnérabilité n'ont pas révélé de crise, car la source du problème ne résidait pas dans les gouvernements en eux-mêmes, ni dans les comptes nationaux. C'est après coup, seulement, que les intermédiaires financiers (privés surtout) "ont été pris en compte dans les engagements visibles de l'État). Donc, pour Krugman (1998), les modèles classiques des crises monétaires n'expliquent pas suffisamment la crise; celle-ci tient avant tout selon lui aux mauvaises pratiques bancaires et à leurs conséquences et, à titre accessoire seulement, aux monnaies. ${ }^{28}$

I1 faut tenir compte d'autres facteurs pour effectuer une analyse éclairée de la crise est-asiatique :

1) les crises financières produisent de très graves effets sur la croissance car elles empêchent l'intermédiation financière d'apporter une contribution financière positive ;

2) les crises est-asiatiques ont résulté d'investissements non seulement excessifs, mais aussi risqués ;

Les cycles d'alternance d'essor et d'effondrement de l'activité provoqués par les excès financiers ont précédé les crises monétaires car la crise financière était le véritable moteur de tout le processus, alors que les fluctuations monétaires en étaient plutôt le symptôme que la cause. La capacité de la crise à se répandre sans de grands chocs exogènes ni d'importants phénomènes de transmission économique peut s'expliquer par le fait que les économies asiatiques touchées étaient ... très vulnérables au pessimisme qui s'autoréalise, capable de provoquer un mouvement de baisse du prix des actifs et de désintermédiation, comme ce fut le cas». 
3) les énormes dépréciations monétaires réelles risquent fort d'entraîner de fortes baisses de la production ;

4) d'autres types de défaillance du marché, notamment le mimétisme, doivent être pris en compte.

Bien que l'analyse proposée dans le présent chapitre ne soit pas compatible avec l'importance accordée par Krugman aux bulles des prix des actifs, aux investissements et aux autres problèmes causés par le risque moral imputable aux garanties implicites de l'État couvrant les intermédiaires financiers peu réglementés, une étude plus pertinente doit aussi envisager plusieurs autres phénomènes, dont :

- les conséquences de la croissance des opérations et de la spéculation monétaires dans le système monétaire international de l'après-Bretton Woods ;

- les raisons pour lesquelles les autorités du Sud-Est asiatique défendent leur quasi-rattachement, face au renforcement du dollar américain, en dépit de ses effets néfastes manifestes sur la compétitivité des exportations et donc sur la croissance ;

- les conséquences de la libéralisation financière, y compris la création de conditions qui ont contribué à l'ampleur des crises ;

- le rôle du mimétisme dans l'exacerbation des crises ;

- les autres facteurs expliquant les effets de contagion.

Un certain nombre de questions de politique économique méritent aussi d'être examinées avec soin, notamment la nature et les conséquences des programmes de «sauvetage» du FMI et des conditionnalités imposées par le Fonds, ainsi que les politiques privilégiées par les milieux financiers internationaux, et non par les milieux nationaux et les autres groupes touchés. Les répercussions de la désintermédiation financière et des monnaies largement sousévaluées pour le développement économique méritent aussi de 
recevoir une attention particulière, du fait, surtout, que la crise menace l'avenir de la croissance et de l'évolution structurelle dans la région, pas seulement de façon directe, mais aussi à titre de conséquence aux ripostes à la crise. Les mesures d'austérité préconisées par le FMI, la communauté financière internationale et d'autres, dont récemment les autorités malaisiennes, risquent fort de jeter le bébé du développement économique avec l'eau du bain de la crise financière.

\section{La crise monétaire de l'Asie du Sud-Est}

A la fin de 1997, Manuel Montes (1998) a publié l'étude qui tente le plus sérieusement de comprendre la crise en Asie du Sud-Est. Il commence par envisager les explications populaires les plus souvent citées, portant à croire que la crise provenait du secteur bancaire, du fait de l'expansion et de la diversification des marchés financiers intérieurs, alimentées par les emprunts privés à court terme. Montes (1998:3) estime que ce constat était particulièrement vrai pour la Thailande, mais moins pour l'Indonésie, la Malaisie et les Philippines (par ordre de pertinence décroissante) et souligne l'importance des effets de contagion; «les différences amènent à se demander jusqu'à quel point la forte dépréciation monétaire (et le désinvestissement de ces économies qui en est résulté) sont sensibles aux fondamentaux économiques».

Dans l'étude de Kaminsky et Reinhart (1996) portant sur 71 crises de balance des paiements et 25 crises bancaires survenues pendant la période $1970-95$, seules trois crises bancaires sont allées de pair avec les 25 crises de balance des paiements qui se sont produites durant la période 1970-79. Cependant, 22 crises bancaires ont coïncidé avec 46 crises des paiements, sur la période 1980-95, ce que les auteurs attribuent à la libéralisation financière engagée à partir des années 80 , accompagnée d'un vif essor du crédit privé, qui a abouti à une crise bancaire, puis à une crise monétaire. Par conséquent, Montes impute la crise monétaire d'Asie du Sud-Est aux 
«libéralisations jumelles» des systèmes financiers intérieurs et des mouvements de capitaux.

Cet analyste affirme que la libéralisation financière a provoqué certains comportements nouveaux dans le système financier, notamment :

1) les institutions financières intérieures disposaient d'une plus grande souplesse et pouvaient offrir des taux d'intérêt leur permettant d'attirer des fonds au niveau national et de rivaliser pour la collecte de fonds étrangers ;

2) elles sont devenues moins dépendantes des prêts à l'État ;

3) la réglementation, tels que les règles et les plafonds d'allocation du crédit, a été revue ;

4) du fait de la concurrence intérieure accrue, pour progresser, il faut élargir les portefeuilles de prêts, souvent en renonçant à la prudence.

Dans le même temps, la libéralisation des mouvements de capitaux a essentiellement garanti la facilité de sortie aux non-résidents, ainsi qu'une réduction des limites imposées sur la détention d'actifs étrangers par les ressortissants, ce qui a involontairement rendu l'exode des capitaux plus aisé.

Montes identifie ensuite les éléments suivants qui constituent les fondamentaux déterminants des économies du Sud-Est asiatique touchées par la crise :

1) la viabilité des systèmes financiers intérieurs ${ }^{29}$;

${ }^{29}$ Montes souligne que l'opinion du marché peut exercer une influence favorable ou défavorable sur les fondamentaux et la santé des systèmes financiers; en particulier, l'effondrement des monnaies d'Asie du Sud-Est imputable au sentiment du marché nuirait à la viabilité des investissements réalisés dans des conditions de change différentes, ce qui pourrait par ailleurs exacerber la crise bancaire intérieure. 
2) la sensibilité de la production intérieure et des exportations aux dévaluations nominales ${ }^{30}$;

3) la viabilité des déficits courants ${ }^{31}$;

4) des taux d'épargne élevés et des finances publiques robustes.

En dépit de la situation budgétaire saine avant la crise, on attend maintenant des économies du Sud-Est asiatique qu'elles dégagent des excédents budgétaires encore plus élevés, malgré le besoin d'un financement public accru des infrastructures physiques èt des services sociaux. Pour rétablir la confiance dans leur monnaie, on leur demande de comprimer leur déficit courant, outre les dépenses publiques, ce qui ne présage rien de bon pour la reprise économique et la viabilité.

Reconnaissant des perspectives limitées mais néanmoins significatives d'indépendance monétaire pour les économies du SudEst asiatique, Montes soutient qu'on ne peut laisser la libéralisation économique compromettre un développement sain du système

${ }^{30}$ Selon Montes, les économies rurales d'Asie du Sud-Est ont été mieux en mesure d'effectuer des dévaluations réelles, à partir de variations nominales du taux de change, auxquelles leur secteur des exportations a pu réagir car il n'était pas entravé par des rigidités de l'offre. Après avoir affirmé que les marchés boursiers ont servi à partager les risques entre les détenteurs d'actifs, au lieu de mobiliser un financement, Montes soutient qu'exception faite des déficiences du système financier, les secteurs réels d'Asie du Sud-Est ont été relativement épargnés par la récente frénésie des marchés des actifs.

${ }^{31}$ Montes fait observer que les prises de participation et les investissements de portefeuille ont dépassé les investissements directs, les prêts et les crédits commerciaux, à titre de mécanismes de financement extene pendant les années 90 . L'auteur cite l'avertissement lancé par Reisen (Montes 1998:34), qui estime qu'il faut résister aux offres de financement étranger si elles «entrainent une appréciation insoutenable de la monnaie, une prise de risques excessive dans le système bancaire et une vive chule de l'épargne privée». En conséquence, dans un monde régi par l'opinion du marché, les monnaies deviennent trop fortes, par suite des offres d'un solide financement externe, et trop faibles lorsque les capitaux refluent. 
financier et les améliorations de la productivité de l'investissement. En guise d'avertissement, il déclare que la solidité des données macroéconomiques fondamentales ne saurait mettre un pays à l'abri de la contagion et de la crise. L'étendue de l'indépendance monétaire dépend en partie d'une saine gestion macroéconomique et de la volonté politique. Partisan des taux de change flexibles, il met en garde contre l'inefficacité des contrôles des capitaux et d'autres efforts en vue de soutenir une monnaie subissant des attaques, qui en réalité, ne font que subventionner davantage des actions spéculatives. La coopération et la coordination internationales ont souvent constitué la meilleure riposte dans de telles circonstances, mais importent également pour engager des initiatives prudentiellès et réglementaires efficaces et réduire «l'arbitrage entre les politiques». Montes recommande aussi de protéger le système bancaire intérieur contre l'instabilité à court terme en établissant des mesures réglementaires et des contrôles des capitaux, et en durcissant la réglementation prudentielle applicable dans la région.

\section{Une réforme internationale?}

Le défi est immense au niveau international, compte tenu en particulier des intérêts acquis sous-tendant les positions américaines et européennes sur la réforme systémique. Ailleurs aussi, toutefois, le système financier international suscite de nombreuses craintes, en raison de sa nature et de son instabilité, dont certains aspects provoquent un regain d'appréhensions à l'occasion de chaque crise nouvelle. Les pays d'Asie du Sud-Est doivent trouver des alliés partageant les mêmes conceptions et s'inspirer des critiques fertiles qui ont été formulées au fil des ans pour élaborer des propositions de réforme susceptibles de recueillir un vaste soutien international.

Fait incroyable, lors de l'assemblée annuelle du FMI et de la Banque mondiale tenue à Hong Kong en septembre 1997, le Comité intérimaire, organe de décision du FMI, qui représente l'ensemble des 181 pays membres du FMI par l'entremise de vingt-quatre ministres, 
a donné au FMI le mandạt de modifier ses statuts, afin d'étendre les «attributions» relatives aux mouvements de capitaux et au compte des transactions courantes de la balance des paiements des pays membres, qu'il possède depuis de nombreuses décennies. ${ }^{32}$

En décembre 1997, l'Organisation mondiale du commerce a également conclu un accord sur les services financiers, qui engage pour l'essentiel les pays membres à amorcer une libéralisation accélérée du commerce des services financiers, selon un calendrier prévu. Le Wall Street Journal a noté que l'accord bénéficierait avant tout aux EtatsUnis et à l'Europe, puisqu'il est tout à fait improbable que le Sud soit en mesure d'exporter des services financiers vers le Nord. Les pays du Sud seront probablement confrontés à des problèmes encore plus graves de balance des paiements, à mesure que leurs services, et donc leur déficit courant, se détérioreront. Une grande partie des services financiers naissants, qui ont émergé sous la protection de l'Etat dans ces pays, risque fort de disparaître sous l'effet de la concurrence internationale livrée par des géants transnationaux profitant d'économies d'échelle et d'autres avantages.

Comme le portent à croire les récentes analyses du bilan et des capacités du FMI parues dans la presse, on constate à l'échelle internationale un scepticisme croissant à propos du rôle de cette

32 Je tiens à remercier Anthony Rowley pour avoir obtenu confirmation de ces faits auprès de Kunio Saito, directeur du nouveau bureau du représentant régional du FMI à Tokyo, le 17 décembre 1997. Le conseil d'administration du FMI tient actuellement une série de réunions pour examiner en détail l'exécution de ce mandat et rendra compte au Comité intérimaire du fonctionnement de ce mécanisme lors de sa réunion de printemps. Par la suite, chacun des gouvernements des pays membres devra ratifier l'amendement, mais la majorité simple súffira. Autrement dit, il ne sera pas nécessaire que le vote soit unanime pour adopter l'amendement aux statuts du FMI. Cependant, d'autres collègues, y compris le professeur Gerald Helleiner, de l'université de Toronto et M. Yilmaz Akyuz de la CNUCED, laissent entendre que la situation n'est pas aussi grave que ne le porte à croire le compte rendu ci-dessus, car le processus d'adoption est beaucoup plus complexe. 
organisation et de ses remèdes pour la crise Est-asiatique en cours. Peut-être en partie par la force de l'habitude, car le FMI est appelé à résoudre des situations en Amérique latine, en Afrique, en Europe de l'Est ou ailleurs, où les déficits budgétaires figurent parmi les problèmes, ses récentes interventions en Asie de l'Est semblent reposer sur la même thérapeutique (indifférenciée).

Un grand nombre de ses programmes ont effectivement un effet récessif et ne se soucient guère des répercussions sociales et autres de ses remèdes. Par conséquent, ce qui est au départ une crise monétaire ou financière aboutit à un ralentissement économique, voire à une récession, en partie à cause de la riposte recommandée par le FMI. Par exemple, bien que toutes les économies est-asiatiques touchées aient enregistré des excédents budgétaires au cours des dernières années (sauf l'Indonésie qui accusait un faible déficit en 1996), le FMI a contraint tous les gouvernements à amputer les dépenses publiques et à accroître leurs excédents budgétaires.

Nombreux sont ceux qui doutent que Ie FMI ait véritablement reconnu les éléments nouveaux de la crise et leurs conséquences («de vieux remèdes pour un nouveau mal»), surtout au début. L'apparente incapacité de cette organisation à prévoir la crise actuelle (dans ses rapports en général enthousiastes sur la région) et à contenir cette dernière ou encore moins à l'inverser, en dépit d'interventions en Thaïlande, en Indonésie et en Corée-n'ont certainement pas inspiré confiance, ni le fait que, alors que les Philippines appliquaient un programme du FMI, ce pays n'ait pas été épargné par la contagion..$^{33}$

Le FMI ne paraît pas tenir suffisamment compte des éléments subjectifs inhérents à la crise et semble la traiter comme si elle était

${ }^{33}$ On peut dire que la chute de la monnaie des Philippines n'a pas été si forte, en partie grâce au fait que les normes bancaires et comptables de ce pays (héritées de la période coloniale) sont jugées meilleures par comparaison, mais aussi parce que les entrées de capitaux à court terme ont été relativement inférieures, étant donné le caractère récent de la reprise économique. 
uniquement imputable à des déficiences macroéconomiques ou autres. Par exemple, en fermant des banques en Indonésie, le FMI a sapé le peu de confiance qui restait, entraînant par là-même une panique généralisée. En outre, alors que le Fonds demande avec insistance que les gouvernements en crise recevant ses concours et les autres pays appliquant ses programmes affichent une transparence accrue, son fonctionnement demeure secret.

L'application de deux poids deux mesures par le FMI, dont témoigne la priorité qu'il paraît accorder à la protection des intérêts des banques et des gouvernements étrangers, a également compromis son rôle officiel d'organisation impartiale cuvrant dans l'intérêt de l'économie d'accueil. Le fardeau des programmes du FMI incombe invariablement au secteur financier intérieur et, en fïn de compte, à l'ensemble de la populationpar le biais des coûts sociaux de la riposte des pouvoirs publics, qui comporte d'ordinaire le sauvetage d'une grande partie du secteur financier, voire plus généralement du secteur des entreprises-qui supporte donc la plupart des coûts de l'ajustement et des réformes, alors que les engagements envers les banques étrangères sont invariablement honorés, bien que les établissements étrangers et nationaux puissent s'être montrés tout autant irresponsables ou imprudents dans leurs pratiques de prêt.

Comme l'a noté en janvier 1998 la Banque des règlements internationaux (BIRD) dans son rapport intitulé Report on the Maturity and Nationality of International Bank Lending (Raghavan 1998 ; Vadarajan 1998), «en dépit de tensions croissantes en Asie du Sud-Est, les prêts bancaires totaux accordés aux pays en développement asiatiques n'ont présenté aucun signe de fléchissement au premier semestre de 1997» (traduction libre). Sur l'année écoulée entre le milieu de 1996 et de 1997, la Corée du Sud a reçu 15 milliards de dollars américain au titre de nouveaux prêts et 1'Indonésie 9 milliards de dollars américains. Les crédits à court terme ont continué de prédominer, puisque 70 pour cent des prêts étaient assortis d'une échéance d'un an, alors que la part des prêts à 
des emprunteurs non bancaires privés a atteint 45 pour cent à la fin de juin 1997. Les banques s'employaient en outre activement à acquérir des «actifs non traditionnels» dans la région (sur les marchés monétaires locaux aux rendements plus élevés) ainsi que d'autres titres de créance. La plupart de ces prêts étaient accordés par des banques du Japon et d'Europe continentale.

Ainsi, les banques occidentales sortiront de la crise non seulement relativement indemnes, mais aussi relativement plus fortes. Certaines banques d'affaires et d'autres institutions financières pourront de surcroît toucher des commissions lucratives grâce au négoce de titres de créance garantis par l'État, alors que les emprunts privés, qui ont précipité la crise, sont convertis en obligations à long terme garanties par l'Etat, dans le cadre des programmes du FMI. Par conséquent, les programmes de sauvetage sont principalement destinés aux banques étrangères, plutôt qu'aux économies ou au peuple est-asiatiques.

Le peu d'empressement des Etats-Unis à contribuer aux opérations de sauvetage organisées par le FMI en Thaïlande, en Indonésie et en Corée du Sud, qui dépassent maintenant cent milliards de dollars américains, traduit aussi les nouvelles priorités américaines, dans le contexte de 1'après-guerre froide. En dépit de sa volonté d'engager davantage de ressources, l'administration américaine a par ailleurs bloqué les initiatives du Japon et d'autres propositions régionales en vue de créer une facilité régionale, par crainte qu'elles ne renforcent le rôle et la prédominance du Japon dans la région et n'affaiblissent la position des États-Unis. Néanmoins, après la panique qui s'est emparée des marchés boursiers du monde entier en octobre 1997, l'administration américaine paraît avoir assumé un rôle prépondérant, en dépit de l'engagement limité des banques américaines dans la région. Il semble que ce changement de position découle des préoccupations des États-Unis àll'égard d'une éventuelle débâcle financière mondiale, de la fonction de principale monnaie de réserve remplie par le dollar américain, et des occasions, 
pour les banques américaines et les autres investisseurs, de tirer profit de la situation.

Il est en général admis que l'intervention du FMI, menée presque parallèlement à la libéralisation financière, a compromis et limité la souveraineté économique nationale. ${ }^{34}$ Un exemple particulièrement accablant en est l'abus manifeste des conditionnalités imposées par le FMI dans le cadre du programme d'aide à la Corée, pour résoudre les questions en suspens en faveur des intérêts américains et japonais (Chossudowsky 1998). La législation et les autres règlements nouveaux qui permettent d'accroître les participations étrangères dans l'économie coréenne et d'en faciliter l'accès aux étrangers, sans grand rapport avec la crise ni avec ses causes immédiates, ont été imposés au gouvernement coréen. La poursuite de l'élimination des principales caractéristiques institutionnelles qui ont rendu possible le miracle coréen depuis les années 60 a été encore plus dommageable. Dans le même temps, comme condition de refinancement de la dette coréenne à court terme, les banques japonaises ont réclamé que l'Etat coréen en garantisse le remboursement.

Plus généralement, dans l'ensemble de la région, tout se passe comme si l'on bradait des marchandises légèrement endommagées dans un incendie, alors que les investisseurs étrangers s'accaparent les meilleurs actifs à vil prix. Si l'on admet que du fait de la dépréciation de la monnaie et, plus généralement, de la crise financière, ces actifs sont considérablement soús-évalués au regard des normes internationales, on ne peut prétendre à une amélioration du niveau de vie (Krugman 1998b), puisqu'en toute probabilité, les nouveaux propriétaires étrangers n'ont pas besoin d'être plus efficients pour acquérir massivement ces actifs.

34 Cependant, il peut être très suspect d'invoquer la «souveraineté économique nationale» lorsque celle-ci est manifestement accaparée par des intérêts particuliers. 


\section{Conclusion}

La crise monétaire et financière survenue en Asie du Sud-Est porte à croire que le miracle économique de la région repose sur des fondements vacillants et non viables. La récente croissance enregistrée par la Malaisie et la Thaïlande fait de plus en plus largement appel à des ressources étrangères, à la fois sous forme de capital et de travail. Des investissements limités et des préjugés inopportuns dans la mise en valeur des ressources humaines ont retardé la valorisation de capacités industrielles et technologiques accrues dans l'ensemble de la région. ${ }^{35}$ Les ressources, la richesse et la main-d'œuvre relativement bon marché de l'Asie du Sud-Est ont permis de maintenir des enclaves de production exportant des produits agricoles, forestiers, minéraux et, plus récemment, manufacturés, mais des milieux d'affaires proches du pouvoir se sont accaparés une grande partie de la richesse créé qui avait été conservée, et ont contribué à la croissance en réinvestissant les ressources accaparées et les autres rentes dans les industries de substitution aux importations, le commerce, les services, les services d'utilité publique privatisés et les infrastructures relevant de l'économie intérieure «protégée».

Trois arguments étroitement liés portant sur la libéralisation et la gestion des affaires publiques ont été avancés (Jomo 1998). Premièrement, la libéralisation financière a nui aux institutions et aux

35 Bien que la critique de la faible croissance de la productivité que Krugman a rendue populaire (1994) puisse comporter des failles théoriques et méthodologiques, il ne fait guère de doute que la croissance est-asiatique a en général été stimulée par des taux élevés d'épargne et d'investissement. Même si cette thèse peut donner l'impression que «tout relève de la transpiration et rien de l'inspiration", comme l'ont laissé entendre les critiques de la PTF, la domination des IDE dans les industries à vocation exportatrice soutenant la concurrence internationale semble indiquer le transfert ou l'importation «d'inspiration», sous la forme de nouveaux investissements productifs et des apprentissages technologiques nécessaires pour accomplir les tâches. 
mécanismes de gestion des affaires publiques qui existaient déjà, sans les remplacer par des dispositifs adéquats. Deuxièmement, ce sont les milieux influents qui ont déterminé les mécanismes de gestion intérieure des affaires publiques, y compris ceux qui s'appliquaient au système financier, ou qui en ont abusé à leur profit. Troisièmement, surtout en Thailande, en Malaisie et en Indonésie, en l'absence de dispositifs adéquats de riposte à la crise, les réactions des pouvoirs publics ont été indûment influencées et compromises entre autres par des intérêts acquis.

Par conséquent, on peut utilement résumer les origines de la crise par différents problèmes de gestion des affaires publiques, au niveau international et national. À l'échelle internationale, ces problèmes ont été soulevés par les transformations des marchés financiers, notamment les marchés de capitaux. Les taux de change flexibles et les autres évolutions récentes ont accru l'étendue et le rythme de la spéculation monétaire. L'augmentation des flux internationaux de capitaux d'investissement a aussi contribué à l'instabilité monétaire. Dans la majorité des cas, ces mouvements font intervenir des investissements de portefeuille et risquent donc davantage d'aggraver l'instabilité, tandis que la part des investissements directs étrangers continue de diminuer.

La libéralisation financière a aussi réduit la surveillance des activités financières, y compris les transactions bancaires et la réglementation prudentielle. On a aussi assisté à une hausse marquée des «activités bancaires privées» et à une progression des opérations bancaires transfrontières, avec la multiplication, de par le monde, des centres financiers extraterritoriaux. La dollarisation croissante de l'économie mondiale, notamment des capitaux internationaux, a aussi largement altéré la nature de cette évolution.

La déréglementation des services financiers ainsi que des investissements, dont la libéralisation des mouvements de capitaux, a par ailleurs aussi réduit la surveillance et la gestion nationales des flux financiers, ce qui a créé les conditions propices aux récentes 
crises survenues en Asie du Sud-Est et en Corée du Sud. L'ampleur de la gestion macroéconomique nationale, notamment la gestion monétaire, a été considérablement réduite par les divers aspects de la libéralisation financière. De ce fait, les possibilités d'initiatives visant le développement ou la recherche de rente s'en sont trouvées fortement réduites.

En raison de la diversité des régimes d'Asie de l'Est, il est difficile de se livrer à des généralisations portant sur toute la région. Les observateurs ont été tentés de contraster les économies et les régimes qui ont subi de graves crises depuis le second semestre de 1997--la Thaillande, les Philippines, I'Indonésie, la Malaisie et la Corée du Sud-avec les autres économies est-asiatiques hautement performantes qui ont été épargnées, à savoir le Japon, Taïwan, Hong Kong et Singapour, ainsi que la Chine. Aucune preuve systématique n'indique que la différence réside principalement dans l'étendue de la corruption, de la recherche de rente, de l'intervention de l'État, de la politique industrielle, de l'orientation vers les exportations, de la croissance de la productivité, des investissements directs étrangers ou de la démocratie. Bien que toutes les économies atteintes aient libéralisé les mouvements de capitaux, il se peut qu'il s'agisse d'une condition nécessaire mais non suffisante de la crise. La grande différence parait résider dans le fait que le groupe de pays touchés possédait peu de réserves de change, contrairement à celui des économies épargnées qui détiennent les réserves de change les plus élevées du monde et n'étaient donc pas exposées à une attaque monétaire.

L'ampleur du désalignement des fondamentaux économiques parmi les économies atteintes par la crise a considérablement varié et ne saurait expliquer en soi les débâcles financières, bien qu'elle laisse présager une plus grande vulnérabilité aux attaques monétaires et une plus forte probabilité de panique. Cette crise a souligné l'importance de l'opinion du marché et il n'existe aucune explication convaincante de ce qui s'est produit, en particulier le mimétisme, qui ne tienne pas 
compte de la psychologie du marché. De ce fait, le retour de la confiance doit nécessairement figurer parmi les priorités de tout programme de redressement, ce qui aboutit au dilemme posé par la tentation de raviver la confiance dans une série de mécanismes potentiellement instables, qui peuvent facilement se retourner contre les économies concernées et aller à l'encontre des ambitions de leurs autorités, comme l'ont montré les crises récentes.

Les explications néolibérales du miracle est-asiatique, autrefois hégémoniques, ont été effectivement remises en cause à partir des années 80 (White 1988 ; Amsden 1989) et sont devenues plus complexes (Chang 1994) et plus nuancées (Jomo et al. 1997) au milieu des années 90 . La Banque mondiale, dans sa réponse qui a fait autorité (1993), a affirmé que les conditions politiques, administratives, culturelles et institutionnelles de l'essor du Japon et des nouvelles économies industrielles est-asiatiques de première génération telles que la Corée du Sud, Taïwan, Hong Kong et Singapour étaient si exceptionnelles qu'elles étaient impossibles à reproduire. On suggérait au contraire que les autres pays en développement tentent d'imiter les nouveaux pays industriels (NPI) comme la Malaisie, la Thaïlande et l'Indonésie, qui, selon la Banque mondiale, étaient parvenus à une croissance et à une industrialisation rapides après la libéralisation engagée au milieu des années 80 .

En réaction, d'autres ont fait valoir que le succès des NPI d'Asie du Sud a été, à plusieurs égards, beaucoup plus modeste que celui des NPI est-asiatiques de première génération et que la viabilité de leur croissance, de leur industrialisation et de leur transformation structurelle a par conséquent été beaucoup plus douteuse. D'après Jomo et al. (1997), l'industrialisation rapide tournée vers les exportations qu'a connue le second groupe à partir du milieu des années 80 est en partie attribuable à une conjoncture favorable; en effet, la dépréciation des monnaies d'Asie du Sud-Est a coïncidé avec l'appréciation de la monnaie du Japon et de celles des NPI estasiatiques, et avec une hausse de la production dans ces économies, 
notamment des coûts de main-d' œuvre, et tient aussi à la levée de certaines restrictions aux investissements, qui ont été remplacées par un nouveau régime d'investissement favorisant beaucoup plus l'industrialisation axée sur les exportations.

Un grand nombre de caractéristiques de l'ancien. système ont été conservées, tandis que les «entrepreneurs», faisant preuve de créativité, ont exploité le nouveau cadre réglementaire à leur profit. Toutes ces caractéristiques ont contribué à façonner l'organisation et la structure industrielle de ces économies. Par conséquent, bien que certaines réglementations aient certes renforcé la croissance et la transformation structurelle, souvent en offrant des rentes et des incitations en vue d'encourager les investissements souhaités, d'autres ont aggravé les abus commis par les rentiers. Quoiqu'il soit possible d'opérer une distinction analytique entre bon nombre de tels effets, ces abus étant relativement isolés et réprimés par l'intervention des pouvoirs publics, d'autres risquent d'être beaucoup plus difficiles à distinguer des rentes dont bénéficient les promoteurs du développement.

Les schémas simplistes et les généralisations à outrance ne permettent pas de reconnaître et de différencier ces deux types d'abus. Les réformes de politique économique qui omettront de le faire encourageront à jeter le bébé du développement avec l'eau du bain des abus, ce qui entraînera des conséquences désastreuses sur les ambitions et les projets des promoteurs du développement. Bien entendu, la détermination à réprimer les abus des rentiers dépend, en fin de compte, de l'indépendance du régime à l'égard de tels intérêts, de la «volonté politique» qui en résulte et de la capacité à entreprendre les réformes nécessaires.

Enfin, comme nous le rappellent les historiens de l'économie ou des affaires, les récentes crises survenues en Asie de l'Est ont eu d'importants précurseurs, même dans la région. Malheureusement, le marché, qui est de plus en plus livré à lui-même, n'a ni la mémoire ni la capacité d'acquérir une immunité naturelle. Il appartient donc aux 
responsables de l'élaboration des politiques d'établir les institutions nécessaires et de reconcevoir ou de restructurer les mécanismes institutionnels de la gestion des affaires publiques afin d'empêcher que la tragédie ne devienne une farce.

Tableau 1 : Prêts bancaires internationaux par bançue à la fin de juin 1997 (en milliards de dollars US)

\begin{tabular}{lccccc}
\hline & $\begin{array}{c}\text { Corée } \\
\text { du Sud }\end{array}$ & Thaïlande & Indonésic & Malaisie & $\begin{array}{c}\text { Pays en } \\
\text { développement }\end{array}$ \\
\hline $\begin{array}{l}\text { Total des emprunts } \\
\begin{array}{l}\text { Bancaires } \\
\text { (\%) }\end{array}\end{array}$ & 103,4 & 69,4 & 58,7 & & 744,6 \\
$\begin{array}{l}\text { Privés non bancaires } \\
\text { (\%) }\end{array}$ & 31,7 & 41,3 & 39,7 & & 275,3 \\
$\begin{array}{l}\text { Etat } \\
\text { (\%) }\end{array}$ & 4,4 & 12,0 & 6,5 & & 352,9 \\
$\begin{array}{l}\text { A court terme } \\
\text { Décembre 1996 }\end{array}$ & 67,5 & 45,7 & 34,2 & 11,2 & 115,6 \\
Janvier 1997 & 70,2 & 45,6 & 34,6 & 16,3 & \\
(\%)
\end{tabular}

Source: C. Raghavan, «BIS Banks kept shovelling Funds to Asia, despite Warnings"), Third World Economics, 177:12-13.

Tableau 2 : Engagement des banques de la zone déclarante de la BIRD envers des emprunteurs hors BIRD, à la fin de juin 1997 (en milliards de dollars US)

Total 1054,9

Allemagne

178,2

Japon

172,7

Etats-Unis

131,0

France

100,2

Royaume-Uni

77,8

Pourcentage des emprunteurs non bancaires

$45 \%$

$\left({ }^{*}\right)$ Le présent chapitre est une révision de l'introduction à Jomo (1998). 


\section{Références}

Bello, Walden, 1997, «Addicted to Capital: The Ten-year High and Presentday Withdrawal Trauma of Southeast Asia's Economies», Issues and Letters, Philippine Center for Policy Studies, September-December.

Chin Kok Fay and Jomo K.S., (n.d.), «Financial Liberalisation and Intermediation in Malaysia».

Chossudowsky, Michel, 1998, «The IMF Korea Bailout », Third World Resurgence, 89, January.

Claessens Stijn and Thomas Glaessner, 1997, Are Financial Sector Weaknesses Undermining the East Asian Miracle?, IBRD/World Bank, Washington, D.C., 1997.

Daim, Zainuddin, 1997, «[ Was Taken By Surprise,» Asiaweek, 7/11/97.

Eatwell, John, 1997, «International Financial Liberalisation: The Impact on

World Development», Selected extracts in John Eatwell, International Journal of Technical Cooperation, 3(2), Winter 1997:157-162.

Eatwell, John, 1997, International Financial Liberalisation: The Impact on World Development, Discussion Paper Series, Office of Development Studies, United Nations Development Programme, New York, May.

Fischer, Stanley, 1997, «IMF - The Right Stuff», Financial Times, 17 December.

Gomez, E. T. and Jomo K. S., 1997, Malaysia's Political Economy: Politics, Patronage and Profits, Cambridge University Press, Cambridge, 2nd edn., 1999.

Iwan, J. Azis, 1997, "Currency Crisis in Southeast Asia: The Bubble Finally Bursts», paper presented at the 45th Annual Conference on the Economic Outlook, organised by Research Seminar in Quantitative Economics (RSQE), University of Michigan, USA, 20-21 November. Jomo, K. S. (ed.) 1998, Tigers in Trouble: Financial Governance, Liberalisation and Crises in East Asia, Zed Books, London.

Jomo, K. S. et al., 1997, Southeast Asia's Misunderstood Miracle: Industrial Policy and Economic Development in Thailand, Malaysia and Indonesia, Westview, Boulder, 1997.

Krugman, Paul, 1997, «Bahtulism», Slate Magazine, 14 August.

Krugman, Paul, 1997, «Currency Crises», prepared for NBER Conference, 
October.

Krugman, Paul, 1997, «Whatever Happened to the Asian Miracle?» Fortune, 18 August.

Krugman, Paul, 1998, «What Happened to Asia?», prepared for a conference in Japan, January.

Long, Simon, 1997, «The Limits to Golf: Regional Implications of the Southeast Asian Currency Depreciations of 1997», paper presented at the IISS/CSIS Conference on Political Change and Regional Security in Southeast Asia, Bali, 7-10 December.

Malhotra, Kamal, (n.d.) «Globalisation, Trade and Financial Integration: The Case of Thailand», paper presented at the Social Research Institute, Chulalongkorn University, Bangkok.

Malhotra, Kamal, 1997, «Celebration of 'Miracle' Turns Into Damage Control by IMF», Focus on the Global South, 3 October.

Montes, Manuel, F., 1998, The Currency Crisis in Southeast Asia, Institute of

Southeast Asian Studies (ISEAS), Singapore.

Raghavan, Chakravarthi, 1998, Third World Economics, 16-31 January.

Sachs, Jeffrey, 1997, «Secretive Workings of the IMF Call for Reassessment», New Straits Times, 23/12/97.

Singh, Ajit, (n.d.), «Financial Liberalisation and Globalisation: Implications for Industrial and Industrialising Economies».

Soros, George, 1997, «Avoiding a Breakdown», Financial Times, 31 December. Soros, George, 1998, «Toward a Global Open Society», The Atlantic Monthly, 281(1): 20-32, January.

Vadarajan, 1998, Times of India, 30-31 January.

Wain, Barry, 1997, «Let's Not Bury Asian Values», Asian Wall Street Journal, 56 December.

Woo-Cumings, Meredith, 1997, «Bailing Out or Sinking In?: The IMF and the Korean Financial Crisis», paper presented at the Economic Strategy Institute, 2 December. 